\title{
Signal detection analysis of serial order effects in auditory matching to sample*
}

\author{
DONALD G. DOEHRING \\ McGill University, Montreal, P.Q., Canada
}

Signal detection theory was applied to the analysis of matching-to-sample tasks in which the sample sound was presented in all possible serial positions before, among, and after three choice sounds that varied in intensity. Eight Ss were tested under all conditions, with trials presented to each ear separately. Both false alarm rate and discriminability varied systematically among the choice sounds as a function of the serial position of the sample sound. Although there was a bias toward choosing a sound once removed from the sample, sounds immediately adjacent to the sample were most discriminable. These results were consistent with the types of decay or interference effects that would be predicted by elementary association theories. A significant left-ear advantage in overall accuracy was also found, but there was no interaction of ears and positions for any of the signal detection measures.

In a previous study of auditory oddity and matching to sample (Doehring, 1971), signal detection theory (Green \& Swets, 1966) was used to analyze serial position effects on three-choice sequence tasks, with hit $(\mathrm{H})$ rate calculated as the ratio of correct choices at a given serial position to all possible correct choices of that position, and false alarm (FA) rate as the ratio of incorrect choices of a given position to all possible errors at that position. Discriminability $\left(\mathrm{d}^{\prime}\right)$, which was determined from the $\mathrm{H}$ and FA rates (Elliott, 1964), represented the relative difficulty of a choice at each position independent of response bias (FA rate) for that position. Some correction for response bias is essential for specifying the relative identifiability of the components of a sequence. As an extreme example, a spuriously high $\mathrm{H}$ rate would occur at any serial position the $\mathrm{S}$ selected on every trial.

Correction for response bias did significantly alter the patterns of accuracy found in the first study (Doehring, 1971). On matching-to-sample tasks where the sample was presented first (S-ABC matching) and last (ABC-S matching), the Ss manifested response biases which resulted in patterns of apparent accuracy ( $\mathrm{H}$ rate) that differed from the patterns of discriminability ( $\left.d^{\prime}\right)$ among the choice sounds. The first two choice sounds were equally discriminable for both matching tasks, but a bias toward responding to the second sound resulted in significantly greater accuracy in detecting a matching sound in the second than in the first position. The two tasks differed with respect to the third sound, for which both $\mathrm{H}$ rate and $\mathrm{d}^{\prime}$ were lowest in S-ABC matching and

*The writer wishes to thank Harriet Emerson, Ruth Libman, and Carolyn Cote for assistance in preparation, administration, and analyses of tests; and Bonnie Bartholomeus for helpful comments on the manuscript. This research was supported by Grant MA-1652 from the Medical Research Council of Canada. highest in ABC-S matching. These results demonstrated that signal detection theory can add an important dimension to the study of temporal sequence effects in auditory discrimination, with further research needed to determine the generality of the phenomena that have been observed.

In the present study, the sample sound was presented in all possible serial positions relative to three choice sounds varying in intensity. The interpolation of the sample sound after the first choice sound (A-S-BC matching) and the second choice sound (AB-S-C matching) should indicate whether there were systematic changes in discriminability as the sample sound was moved from the first position (S-ABC matching) to the last position (ABC-S matching). Trials were presented separately to the two ears to determine if a previously observed left-ear advantage (Doehring, 1972) would be found with the present procedure; and two levels of intensity difference were presented to investigate the influence of overall task difficulty on serial position effects relative to sample position.

\section{METHOD}

Eight paid Ss, aged 16 to 24 years, were tested. All had no more than $10 \mathrm{~dB}$ deviation from audiometric zero (ISO) in either ear at $2,000 \mathrm{~Hz}$, and were right-handed in both writing and throwing.

Each of the four matching-to-sample tasks used in this study consisted of three choice sounds, differing in intensity, and a sample sound of the same intensity as one of the choice sounds. The tasks differed only with respect to the serial position of the sample sound, which was presented first in S-ABC matching, second in A-S-BC matching, third in AB-S-C matching, and fourth in ABC-S matching. S pressed one of a horizontal series of pushbuttons to indicate his judgment of the serial position of the matching sound, and was given immediate knowledge of the correct position. The pushbuttons were labeled $\mathrm{A}, \mathrm{B}, \mathrm{C}$, and $\mathrm{S}$, with the position of the $S$ (sample) pushbutton varied according to the task.

Test stimuli were tape-recorded sequences of four $2,000-\mathrm{Hz}$ tones, $1 \mathrm{sec}$ in duration, with onset and decay times of $25 \mathrm{msec}$ and interstimulus intervals of $1 \mathrm{sec}$. The choice sounds were presented at intensities of either 48.6, 50, and $51.4 \mathrm{~dB}(1.4-\mathrm{dB}$ steps) or 48,50 , and $52 \mathrm{~dB}$ (2-dB steps). Testing was done in a sound-treated room. S listened through matched TDH 39 earphones.

Each $S$ received 1,920 test trials. There were an equal number of trials to each ear at each intensity difference for each task. The trials were presented in 16 series, one for each ear at each intensity difference on each task. Each series began with 9 practice trials, followed by 120 test trials. Ss were given eight sessions, on each of which one task was presented to the two ears at one level of intensity difference. The order of tasks and ears was counterbalanced among Ss, with the $2.0-\mathrm{dB}$ difference always presented before the 1.4-dB difference for a given task. The matching sound was presented at each of the three different intensities and in each of the three serial positions on an equal number of trials, with the positions and intensity values of the sounds otherwise randomized. 
Table 1

Mean Hit (H) Rate, False Alarm (FA) Rate, and Discriminability $\left(d^{\prime}\right)$ for the Eight Subjects on Each of the Four Matching to Sample Tasks

\begin{tabular}{lcccc}
\hline & \multicolumn{4}{c}{ Serial } \\
\cline { 2 - 5 } Task & 1 & 2 & 3 & 4 \\
\hline H Rate & & & & \\
S-ABC & Sample & 46 & 55 & 45 \\
A-S-BC & 46 & Sample & 56 & 58 \\
AB-S- & 52 & 52 & Sample & 54 \\
ABC-S & 42 & 54 & 55 & Sample \\
Combined Tasks & 47 & 55 & 53 & \\
FA Rate & & & & \\
S-ABC & Sample & 21 & 32 & 24 \\
A-S-BC & 18 & Sample & 24 & 28 \\
AB-S-C & 26 & 23 & Sample & 21 \\
ABC-S & 21 & 29 & 25 & Sample \\
Combined Tasks & 21 & 27 & 25 & \\
d' & & & & \\
S-ABC & Sample & 74 & 60 & 57 \\
A-S-BC & 82 & Sample & 89 & 80 \\
AB-S-C & 71 & 80 & Sample & 89 \\
ABC-S & 63 & 68 & 80 & Sample \\
Combined Tasks & 72 & 74 & 77 & \\
\hline
\end{tabular}

\section{RESULTS}

The results were analyzed in terms of $\mathrm{H}$ rate, FA rate, and d' among positions, as calculated separately for each ear at each intensity difference on each task. Since the $\dot{F A}$ and $d^{\prime}$ measures were meaningful only for analyses involving position effects, comparisons not involving position effects are reported in terms of $\mathrm{H}$ rate alone. The four tasks did not differ significantly in $\mathrm{H}$ rate, thus demonstrating that the two new matching tasks were not unduly difficult. Overall $\mathrm{H}$ rate was, in fact, .53 for both A-S-BC and AB-S-C matching, as compared with .49 and .50 for $\mathrm{S}-\mathrm{ABC}$ and $\mathrm{ABC}-\mathrm{S}$ matching. As would be expected, accuracy was significantly higher $(F=24.96$, $\mathrm{p}<.01)$ for the $2.0-\mathrm{dB}(.54 \mathrm{H}$ rate) than for the $1.4-\mathrm{dB}$ intensity steps (.48 $\mathrm{H}$ rate). In accordance with previous findings (Doehring, 1972), left-ear accuracy (.55) was significantly higher $(F=28.16, p<.01)$ than right-ear accuracy (.48), with left-ear advantages of $2 \%, 4 \%, 5 \%$, $6 \%, 7 \%, 9 \%, 10 \%$, and $11 \%$ for the eight Ss. There was a significant interaction of tasks and intensity differences $(\mathrm{F}=3.66, \mathrm{p}<.05)$ resulting from a relatively greater advantage of A-S-BC (.58) and AB-S-C (.57) over S-ABC (.51) and ABC-S (.52) matching at the 2.0-dB than at the 1.4-dB intensity steps, where $H$ rate was .49 for A-S-BC, .48 for AB-S-C, .46 for S-ABC, and .49 for ABC-S. This suggests that the intermediate samples are advantageous for relatively simple discrimination tasks. None of the other interactions among tasks, intensity differences, and ears approached significance.

Mean $\mathrm{H}$ rate, $\mathrm{FA}$ rate, and $\mathrm{d}^{\prime}$ at each position on the four tasks are shown in Table 1. There was a significant difference in $\mathrm{H}$ rate among positions for combined tasks $(F=5.24, p<.05)$. Newman-Keuls tests showed that $\mathrm{H}$ rate was significantly lower at the first position than at the second $(p<.05)$ and third $(p<.05)$ positions.
However, there was no significant interaction of positions and tasks, demonstrating that these positional differences did not vary significantly as a function of the serial position of the sample sound. Nor were there significant interactions of positions with intensities or ears.

There was a significant difference in FA rate among positions for combined tasks $(F=4.89, \mathrm{p}<.05)$, with the FA rate for the second position being significantly higher than that of the first $(p<.05)$. There was also a significant interaction of tasks and positions $(F=2.96$, $p<.05$ ). This occurred because FA rate increased from the first to third position for A-S-BC, decreased across positions for AB-S-C, and was lowest at the first position and highest at the second for $\mathrm{S}-\mathrm{ABC}$ and $\mathrm{ABC}-\mathrm{S}$ matching. The interactions of positions with ears and intensities were not significant.

The difference in $\mathrm{d}^{\prime}$ among positions was not significant for combined tasks, but there was a significant Task by Position interaction $(F=4.74$, $p<.01)$. This occurred because $d^{\prime}$ decreased from the first to third positions in $S-A B C$ matching, increased across positions in AB-S-C and ABC-S matching, and was highest for the second position in A-S-BC matching. Among the remaining interactions, there was a significant Task by Interisity by Position interaction ( $F=$ $2.71, p<.05)$ because the positional differences for A-S-BC and AB-S-C matching were much more marked with the $2.0-\mathrm{dB}$ than with the $1.4-\mathrm{dB}$ intensity steps.

\section{DISCUSSION}

Interpolating the sample sound among the choice sounds did not make matching to sample more difficult, but actually somewhat easier for discriminating relatively large intensity differences. If only accuracy of discrimination had been analyzed, it would have appeared that varying the sample position had no effect at all, since there was not a significant Task by Position interaction for $\mathrm{H}$ rate. However, these interactions were significant when the data were analyzed for FA rate and $\mathrm{d}^{\prime}$, demonstrating that variation of sample position did systematically affect both response bias and discriminability among positional choices.

The most simple generalization with regard to the results for FA rate is that the $S$ tends to be biased toward responding to the choice once removed from the sample (Table 1). This trend was also found for S-ABC matching in a previous study with adults (Doehring, 1971) and in three different age groups of children (Doehring \& Libman, 1973). As before, this positional response bias resulted in patterns of $\mathrm{H}$ rate that differed from the patterns of $\mathrm{d}^{\prime}$.

Differences among positions in $\mathrm{d}^{\prime}$ indicate that the choices adjacent to the sample were more discriminable than nonadjacent choices on all four tasks (Table 1). On the tasks where all three sounds preceded (ABC-S) or followed (S-ABC) the sample, the most remote sounds were the least discriminable. Where there were choices both preceding and following the sample (A-S-BC and AB-S-C), the sound following the sample was more discriminable. On these tasks, the greater discriminability of adjacent sounds following as compared with those preceding the sample cannot be readily explained. but could reflect a process analogous to the time-order error observed in classical psychophysical studies (Guilford. 1954). The decreased discriminability of remote sounds on all tasks 
might be explained in terms of either a passive decay of stimulus traces or interference produced by intervening sounds (cf. Kinchla \& Smyzer, 1967: Wickelgren, 1969). These effects might be more clearly delineated by varying the interstimulus intervals and the number of choice sounds. The possible influence of decaying traces in particular would be clarified by determining whether incorrect choices of remote sounds tend to be sounds more intense than the sample. The significant interaction of tasks, intensities, and positions for $\mathrm{d}^{\prime}$ suggests that overall difficulty of detecting differences has some bearing on position effects, since the increased discriminability of sounds following the sample in A-S-BC and AB-S-C matching was much enhanced when intensity differences were large.

The significantly higher $\mathrm{H}$ rate for the left ear provides a further indication that dichotic presentation is not essential for the demonstration of ear asymmetry, and a further confirmation of right-hemisphere dominance for intensity discrimination (Doehring, 1972).

Although any further interpretation of the present findings must be highly speculative, the results do extend our knowledge of the conditions under which changes in auditory sequential processing occur. The previously observed variations in FA and $\mathrm{d}^{\prime}$ for S-ABC and ABC-S matching (Doehring, 1971; Doehring \& Libman, in press) were largely confirmed; and the two additional tasks revealed that the patterns of response bias and discriminability were consistently related to the temporal position of the sample sound. Further research is needed to determine whether nonverbal auditory sequences are indeed identified by a process analogous to that postulated by proponents of elementary association theories (cf. Bryden. 1967) rather than by a more dynamic and holistic process (cf. Lashley, 1951).

\section{REFERENCES}

Bryden, M. P. A model for the sequential organization of behavior. Canadian Journal of Psychology, 1967, 21, 37-56.

Doehring, D. G. Serial order effects in auditory discrimination by oddity and matching to sample. Perception \& Psychophysics, 1971, 10, 137-141.

Doehring, D. G. Ear asymmetry in the discrimination of monaural tonal sequences. Canadian Journal of Psychology, $1972,26,106-110$

Doehring, D. G., \& Libman, R. A. Signal detection analysis of auditory discrimination by children. Perceptual \& Motor Skills, in press.

Elliott, P. B. Tables of d'. In J. A. Swets (Ed.), Signal detection and recognition by human observers. New York: Wiley, 1964.

Green, D. M., \& Swets, J. A. Signal detection theory and psychophysics. New York: Wiley, 1966.

Guilford, J. P. Psychometric methods. (2nd ed.) New York: McGraw-Hill, 1954.

Kinchla, R. A., \& Smyzer, F. A diffusion model of perceptual memory. Perception \& Psychophysics, 1967, 2, 219-229.

Lashley, K. S. The problem of serial order in behavior. In L. A Jeffress (Ed.), Cerebral mechanisms in behavior. New York: Wiley, 1951

Wickelgren, W. A. Associative strength theory of recognition memory for pitch. Journal of Mathematical Psychology, 1969, $6,13-61$.

(Received for publication September 18, 1973.)

\section{Stroop interference with long preexposures of the word: Comparison of pure and mixed preexposure sequences*}

\section{FREDERICK N. DYER $\dagger$ \\ L.S. Army Medical Research Laboratory, Fort Knox, Ky. 40121}

The delay of color naming when the patches are incongruent color names was studied as a function of the duration of a preexposure of the word in black prior to coloration. Individual word and control stimuli were presented in a tachistoscope, and naming latencies were recorded with a voice key. Interference decreased to very low levels at $2 \mathrm{sec}$ of preexposure, then increased to intermediate levels for longer preexposures. Facilitation of color-naming by congruent color names generally paralleled interference effects. Sequences of stimuli in which the preexposure interval varied randomly from trial to trial showed longer color-naming times for short preexposures than did sequences where all stimuli in the sequence used the same preexposure. The utility of this and a related procedure for study of the central activity produced by viewing a written word was discussed.

Color-naming is substantially delayed when the color patches are shaped as words denoting colors different

*The author wishes to thank Dennis M. Buck, who collected the experimental data.

tRequests for reprints should be sent to Frederick N. Dyer, Experimental Psychology Division, U.S. Army Research Laboratory, Fort Knox, Ky. 40121. from the color of the patch (Stroop, 1935). This delay occurs despite instructions to ignore the word, and even extensive practice (Stroop, 1935; Jensen \& Rohwer, 1966) fails to eliminate this very reliable phenomenon. The delay of color-naming is a measure of an involuntary reading response, and provides a useful tool for study of written word stimuli (Dyer, 1973).

Dyer (1971) used the Stroop phenomenon to illustrate changes in the activity associated with a word stimulus during brief viewing periods. Individual words denoting colors were exposed in black for one of several short periods ( 0 to $500 \mathrm{msec}$ ), then the black word was illuminated in a color different from that denoted by the word. Ss were instructed to name the color as soon as possible following coloration of the black word. A series of Vs were exposed in black prior to coloration for a control condition. The difference in response latency between the incongruent name condition and the control Vs condition (Stroop interference) was found to first increase then decrease as a function of increasing duration of the black-word exposure prior to coloration. Black-word preexposures of 20,40 , and $60 \mathrm{msec}$ produced more delay of color-naming than a simultaneous exposure of the word and color ("0" 